\title{
Prenatal exposure to moderate levels of ethanol can have long-lasting effects on learning and memory in adult offspring
}

\author{
ROBERT J. SUTHERLAND \\ University of New Mexico, Albuquerque, New Mexico \\ ROBERT J. McDONALD \\ University of Toronto, Toronto, Ontario, Canada \\ and \\ DANIEL D. SAVAGE \\ University of New Mexico, Albuquerque, New Mexico
}

\begin{abstract}
Prenatal alcohol exposure can produce long-lasting cognitive and learning impairments in children. Previous studies have suggested that prenatal ethanol exposure can produce neurochemical abnormalities in hippocampal formation. We examined whether performance in the Morris water task, known to be sensitive to interference with hippocampal function, would be affected in adult offspring of rat dams who consumed moderate levels of ethanol through pregnancy. Rat dams consumed one of three diets throughout gestation: (1) Bio-Serve liquid diet containing $5 \%$ ethanol (v/v; 26\% EDC), which produces a maternal peak blood ethanol concentration of $100 \mathrm{mg} / \mathrm{dl}$; (2) pair fed an isocalorically equivalent amount of $0 \%$ ethanol liquid diet; or (3) laboratory chow ad libitum. Adult offspring from each of the three maternal diet conditions were trained in one of three versions of the Morris water task. We employed the "standard" fixed hidden platform task, the moving platform task, and a task that measures competition between cue and place navigation. All three groups learned to navigate normally in the fixed hidden platform version and showed clear preferences for the goal location during no-platform probe trials. In contrast, in the moving platform version and the cue versus place competition task, the prenatally exposed rats were abnormal relative to the rats of the other two maternal diet conditions. In both of these tasks, they behaved in a manner consistent with the idea that acquisition of new place information does not proceed as effectively as in normal rats. These data are consistent with the hypothesis that hippocampal circuitry and neurochemistry related to synaptic plasticity are important targets underlying at least some of the long-lasting deleterious cognitive effects of prenatal alcohol exposure.
\end{abstract}

Fetal alcohol syndrome (FAS; Clarren \& Smith, 1978; Jones \& Smith, 1973; Jones, Smith, Ulleland, \& Streissguth, 1973; Lemoine, Haronsseau, Borteryu, \& Menuet, 1968 ) is a set of major morphological and neurological abnormalities in offspring exposed to high levels of ethanol in utero. However, moderate ethanol consumption during pregnancy is much more prevalent, with estimates ranging from $5 \%$ to $20 \%$ of pregnant women. The number of children exposed prenatally to moderate levels of

This research was supported by a grant from the NSERC of Canada (to R.J.S. \& R.J.M.), NIH Grants AA1 1333 and AA06548 (to R.J.S. and D.D.S., respectively), Grant RR08139 from the Minority Biomedical Research Support Program (to D.D.S.), and a grant from the Dedicated Health Research Fund of the University of New Mexico School of Medicine (to D.D.S.). The authors thank Linda Paxton, Christian Sanchez, Samantha Lopez, Ana Polaco, Laura Cruz, Jeremy Hanlon, and Lorina Duran for their work on the fetal ethanol exposure paradigm. The authors also thank Lisa Mumby and Brenton Cooper for their participation in the behavioral testing. Correspondence should be addressed to R. J. Sutherland, Department of Psychology, Logan Hall, University of New Mexico, Albuquerque, NM 87131-1161 (e-mail: sutherla@unm.edu). ethanol may be 10 to 20 times higher than the number exposed to the heavy or binge patterns of consumption associated with FAS (Centers for Disease Control \& Prevention, 1994).

According to clinical evidence, moderate ethanol consumption can cause subtle, long-term impairments in the absence of gross morphological or generalized neurological defects associated with FAS (Abel, 1984; Hanson, Streissguth, \& Smith, 1978; Jacobson, Jacobson, \& Sokol, 1994; Jacobson et al., 1993; Mattson, Riley, Gramling, Delis, \& Jones, 1997; Shaywitz, Cohen, \& Shaywitz, 1980). Most striking is the impression that moderate ethanol exposure in utero causes cognitive deficits that may not become apparent until the child is challenged during the educational years (Conry, 1990; Streissguth, Barr, \& Sampson, 1990) and may increase in severity as the child matures (Streissguth et al., 1991). Recently, the Institute of Medicine recommended expanding the classification of prenatal alcohol-related effects to include a new category called alcohol-related neurodevelopmental disorders (ARND). Symptoms of ARND involve behav- 
ioral or cognitive abnormalities, including learning difficulties, deficits in school performance, poor capacity for abstraction, and problems in memory, attention, or judgment.

The present experiment was directed toward developing a better understanding of prenatal ethanol effects on adult learning and memory and toward developing reliable behavioral measures sensitive to moderate prenatal ethanol exposure in rats. Although there is no consensus on the neuroanatomical or neurochemical loci of fetal alcohol effects on cognition, attention has been directed toward the hippocampal formation as one site among many where disrupted neural development could produce important, long-lasting behavioral consequences. Beginning with the work of Brenda Milner with human amnesic patients several decades ago (Milner, 1965), the hippocampal formation has been a central focus of research on the neuroanatomical bases of learning and memory. Work with humans with highly selective hippocampal damage (Zola-Morgan, Squire, \& Amaral, 1986) and experiments with rats and monkeys using selective hippocampal lesions show that hippocampal damage alone is sufficient to produce profound and enduring impairment of certain forms of learning and memory (Squire, 1992). Learning and remembering spatial and other forms of environmental relationships are particularly affected.

The neurobiological implications of the subtle, but perhaps more pervasive, effects of moderate ethanol consumption during pregnancy on cognitive processes in affected offspring prompted our earlier investigation of the effects of moderate ethanol consumption on glutamatergic neurotransmission and synaptic plasticity (Savage, Montano, Otero, \& Paxton, 1991). In our present fetal ethanol exposure paradigm, rat dams receive a $5 \%(\mathrm{v} / \mathrm{v})$ ethanol diet, an isocalorically equivalent diet, or rat chow ad libitum. Importantly, this moderate ethanol exposure does not affect birth weight, litter size, neonatal mortality, offspring growth curves, or whole brain weight, relative to the control groups.

Despite this apparent normality, neurochemical observations in these rats reveal changes in at least four subtypes of amino acid receptors and at least several of the enzymes (Allan, Caldwell, \& Savage, 1997; Allan, Wu, Paxton, \& Savage, 1998; Farr, Montano, Paxton, \& Savage, 1988; Queen, Sanchez, Lopez, Paxton, \& Savage, 1993; Savage et al., 1991) in hippocampal formation that may be responsible for some functional abnormalities, and at least some of these changes are at loci critical for normal long-term potentiation (LTP). Finally, we have found with our fetal alcohol exposure paradigm that the majority of exposed rats in adulthood show marked decreases in induction and maintenance of LTP at perforant path-dentate synapses (Sutherland, McDonald, \& Savage, 1997). Our present working hypothesis is that prenatal ethanol exposure causes multiple neurochemical changes leading to synaptic plasticity deficits within the hippocampal formation and possibly other re- gions of cortex. These changes, which manifest as LTP deficits, have subtle behavioral ramifications that are most apparent in more challenging, relevant learning tasks.

Given the potential importance in understanding neurobiological and cognitive/behavioral consequences of prenatal ethanol exposure, we sought to extend observations of the effects of moderate prenatal ethanol levels on hippocampal LTP and changes in hippocampal neurochemical processes by studying the behavior of adult offspring in a behavioral situation, the Morris water task, which has repeatedly been shown to be sensitive to manipulation of hippocampal function. This Morris water task is especially well suited to extending our understanding of the behavioral deficit produced by fetal alcohol exposure for several reasons. Prior work has shown that acquisition of place information in this task is sensitive to various types of hippocampal disruption. These include nonspecific lesions, such as hippocampal aspiration, electrolytic damage, and transections of fornix or perforant pathway (Morris, Garrud, Rawlins, \& O'Keefe, 1982; Skelton \& McNamara, 1992; Sutherland, Kolb, \& Whishaw, 1982; Sutherland \& Rodriguez, 1989; Sutherland, Whishaw, \& Kolb, 1983), as well as very selective subtotal lesions involving each of the major hippocampal subfields, $\mathrm{CA}_{1}$, $\mathrm{CA}_{3}$, and components of the dentate gyrus (Auer, Jensen, \& Whishaw, 1989; Sutherland, Hoesing, \& Whishaw, 1990; Sutherland et al., 1983; Whishaw, 1987). Furthermore, certain manipulations of hippocampal synaptic plasticity (Castro, Silbert, McNaughton, \& Barnes, 1989; Morris, Anderson, Lynch, \& Baudry, 1986) have been shown to disrupt acquisition of Morris water task performance (but see Sutherland, Dringenberg, \& Hoesing, 1993).

Several experiments have addressed the issue of whether prenatal or early postnatal exposure to alcohol affects Morris water task performance of rats. Gianoulakis (1990) showed that prenatal alcohol exposure produced a deficit in Morris water task performance at 40 , 60 , and 90 days of age, although there was clear evidence for some place learning on the part of the FAE rats. Blanchard, Pilati, and Hannigan (1990) and Blanchard, Riley, and Hannigan (1987) found a deficit after prenatal alcohol exposure in 21-day-old rats, which was not present by Postnatal Day 24. The reason for the difference between the results of the Gianoulakis study and the Blanchard et al. (1987) studies is uncertain - there were several potentially important procedural differences.

In the present experiments, we addressed the issue of whether moderate prenatal alcohol exposure produces deficits in performance in adult offspring further, using three versions of the Morris water task. The versions differ in the extent to which there is a demand placed on rapid learning of new location information or to which there is interference from non-place-learning strategies. First, we tested the effects of prenatal exposure to moderate levels of alcohol on acquisition of the "standard" version, involving a single fixed hidden platform location (Morris, 1981). In a second version, we took advantage of a procedure developed by Whishaw $(1985,1987)$ in 
which the hidden platform location is changed each day but remains the same for all trials on a given day. After training in this task, normal rats show essentially one trial place learning each day they are tested. Whishaw and colleagues have shown that his procedure is exquisitely sensitive, even to partial damage in one of the hippocampal subfields (Auer et al., 1989). In a third version, rats are trained to swim to a visible landmark in a fixed location in the pool. On one trial after training, the visible landmark is moved to a new location, and the rat's preference for swimming to the landmark versus swimming to the location is recorded. McDonald and White (1994) showed that approximately half of normal rats prefer to swim directly to the landmark but that forebrain damage can differentially affect this proportion. Whereas nearly all rats with hippocampal damage prefer to swim directly to the landmark, nearly all rats with striatal damage swim to the old location, ignoring the landmark. On our view of the special sensitivity of hippocampal circuitry to teratogenic effects of prenatal alcohol exposure, we predict the exposed rats will show a strong preference for swimming directly to the landmark.

\section{METHOD}

\section{Prenatal Ethanol Exposure Paradigm}

Five-month-old Sprague-Dawley rat dams (Harlan Industries, Indianapolis) were individually housed in plastic cages in a temperaturecontrolled room on a 16:8-h dark:light schedule (lights off from 1730 to $0930 \mathrm{~h}$ ) in the University of New Mexico Medical School vivarium. Beginning on Day 1 of gestation, rat dams were placed in one of three diet groups. Two of the three diets consisted of a BioServ liquid diet (Frenchtown, NJ). One group received liquid diet containing 5\% ethanol ( $\mathrm{vol} / \mathrm{vol} ; 26 \%$ ethanol-derived calories). This group received $110 \mathrm{ml}$ of $5 \%$ ethanol liquid diet at $1730 \mathrm{~h}$ each day. The feeding tubes were removed $16 \mathrm{~h}$ later (at $0930 \mathrm{~h}$ on the following morning), and water bottles were placed on the cages. The other liquid diet group, serving as pair-fed control, was given a $0 \%$ ethanol liquid diet (isocalorically equivalent to the $5 \%$ ethanol diet) for $16 \mathrm{~h}$ each day. A third diet group received Purina breeder block chow ad libitum and served as control for the paired feeding technique. Rat dams were maintained on these diets throughout gestation. Maternal blood ethanol concentrations produced by consumption of the $5 \%$ ethanol liquid diet were determined in a separate set of dams using methods described previously (Sutherland et al., 1997).

At birth, all litters were weighed, culled to 10 pups, and crossfostered onto surrogate untreated dams. Rat pups were weaned at 30 days of age and maintained on lab chow and water ad libitum. Groups were composed of offspring from at least three separate litters from each of the three diet groups.

Eighty-seven male Sprague-Dawley offspring were used in behavioral testing. Thirty-two rats served in Task 1, 19 rats in Task 2, and 36 rats in Task 3. After transfer from the Medical School Animal Resource Facility, they were housed individually in the Department of Psychology vivarium at the University of New Mexico and were maintained on a 12:12-h light:dark cycle. Water and a standard laboratory rodent diet were continually available. At the time of behavioral testing, the rats weighed between 300 and $450 \mathrm{~g}$ and were between 100 and 200 days of age. There were no group age or weight differences at the time of testing.

\section{Apparatus}

A circular, white swimming pool ( $1.4 \mathrm{~m}$ diameter, $0.8 \mathrm{~m}$ height $)$ was used. It was filled with water $\left(20^{\circ} \mathrm{C}\right)$ rendered opaque by dissolving approximately $1,500 \mathrm{ml}$ of instant skim milk powder. The pool was located in a room with many clearly visible, distinctive cues on the walls and ceiling. In Tasks 1 and 2, a clear Plexiglas platform $(13 \times 13 \mathrm{~cm})$ was positioned in the pool, with its surface $1.5 \mathrm{~cm}$ below the water surface. For most of the trials in Task 3 , a black wooden platform $(13 \times 13 \mathrm{~cm})$ covered with a metal mesh was positioned in the pool, with its top protruding $2 \mathrm{~cm}$ above the water surface. For the remaining trials (12) in Task 3, the Plexiglas platform was used. Behavior during each trial was recorded using an automated, computer-based, video tracking system (HVS Image Analysis, VP112). The measures included time to find the platform, swim path length, and proportion of swim distance in each quadrant.

\section{Morris Water Task Procedures}

Task 1: Fixed hidden platform task. The rats from the three maternal diet conditions, ad-lib $(n=11)$, pair-fed $(n=10)$, and $5 \%$ ethanol $(n=11)$, were trained. They received 8 trials each day for 5 days. The rats were released once from each of the cardinal compass directions at the pool's perimeter in each block of four trials. The maximum trial duration was $90 \mathrm{sec}$. If the rat was still swimming after $90 \mathrm{sec}$, it was removed from the pool and placed by hand on the platform for $10 \mathrm{sec}$. Typically, 5-7 min elapsed between trials. The hidden platform was positioned in the center of the northwest quadrant of the pool for all rats. On the last day of training, all rats received one trial lasting $20 \mathrm{sec}$ with the platform removed from the pool to test for searching preference. On this probe trial, the proportion of swim path length in the four quadrants was analyzed.

Task 2: Moving hidden platform task. A different set of rats from the three maternal diet conditions, ad-lib $(n=6)$, pair-fed $(n=6)$, and $5 \%$ ethanol $(n=7)$, was trained for 13 days in the moving hidden platform version of the Morris water task (Whishaw, 1987). The rats received 8 trials each day as described above. There was a $90-\mathrm{sec}$ trial maximum; if the rat did not find the platform, it was placed by hand on the platform for $10 \mathrm{sec}$. Each day the platform was moved to a new position according to a pseudo-random sequence; however, for all trials in a given day, the platform remained in the same position. The platform was never positioned in the exact center of the pool, nor was it positioned closer than $5 \mathrm{~cm}$ to the pool wall. Furthermore, the center of the platform was never positioned within $5 \mathrm{~cm}$ of a previously selected position. After the last trial of training on the last day of training, all rats received a 20 sec probe trial with the platform removed, and the proportion of swimming in the four quadrants was analyzed.

Task 3: Cue - place competition task. The rats from the three maternal diet conditions, ad-lib $(n=12)$, pair-fed $(n=10)$, and $5 \%$ ethanol $(n=14)$, were trained for 12 days in the visible platform task version of the Morris water task. All of the rats were trained to swim to a visible platform located in the center of the northwest quadrant of the pool. There were 4 trials each day, and each rat was released from the four different starting points once per day. A trial ended when the rat climbed onto the platform or when $30 \mathrm{sec}$ had elapsed. If a rat was still swimming at the end of a trial, it was placed by hand on the platform for 10 sec. After 3 days of testing (12 trials), each rat received 4 trials (the next day) during which the visible platform was replaced with the invisible platform in the same location. This sequence of 12 visible platform trials followed by 4 hidden platform trials was repeated three times, until 36 visible platform trials and 12 invisible trials had been completed. On the final day of testing (Day 13, competition test), the visible platform was moved to the center of the diagonally located quadrant. There was no platform located in the original location. Each rat received a single swim trial from a start location on the pool's perimeter that 
was equidistant from the visible platform and the previous goal location. We used only one swim trial because subsequent trials would be affected by new learning.

\section{RESULTS}

\section{Fetal Ethanol Exposure Paradigm}

Table 1 summarizes rat dam caloric intake, ethanol consumption, and maternal blood ethanol concentration data, along with the impact of these diets on litter size and offspring birth weight. The average daily consumption of ethanol by rat dams on the $5 \%$ ethanol liquid diet was $13.1 \mathrm{~g} \mathrm{EtOH} / \mathrm{kg}$ body weight/day. Litter size and pup birth weights were not different between the pair-fed group and the 5\% ethanol liquid diet group. Furthermore, no gross anatomical abnormalities were noted at birth in the fetal-ethanol-exposed rats, nor were there any differences among the three diet treatment groups in brain weights of the adult offspring at the time of sacrifice (data not shown).

\section{Behavioral Results}

Task 1: Fixed hidden platform task. All rats showed improvement in escape latency (see Figure 1). Although there was a trend for the rats in the fetal-alcohol-exposed group to display longer latencies, the main effect of maternal diet did not reach statistical significance $[F(2,29)=$ $2.1, p<.1]$. The improvement across trials was statistically significant $[F(39,1131)=33.5, p<.001]$, and the interaction between maternal diet and trials was not statistically significant $(F<1.0)$. Given the age range of rats in the study, we calculated a correlation between age at testing and mean latency to find the platform across all trials. The observed correlation $(r=.12)$ was not statistically significant $(p>.4)$. Consistent with this lack of effect on time to find the platform, the proportion of swimming distance in the correct quadrant on the noplatform probe trial did not differ between the groups $[F(2,29)=1.2, p<.5]$. The observed swim preferences were $52.4 \%, 61.1 \%$, and $55.7 \%$ for the ad-lib, pair-fed, fetal-alcohol-exposed groups, respectively.

Task 2: Moving hidden platform task. All rats showed clear evidence of improvement in time to find the platform. However, the fetal-alcohol-exposed rats clearly showed a smaller improvement in latency over the first

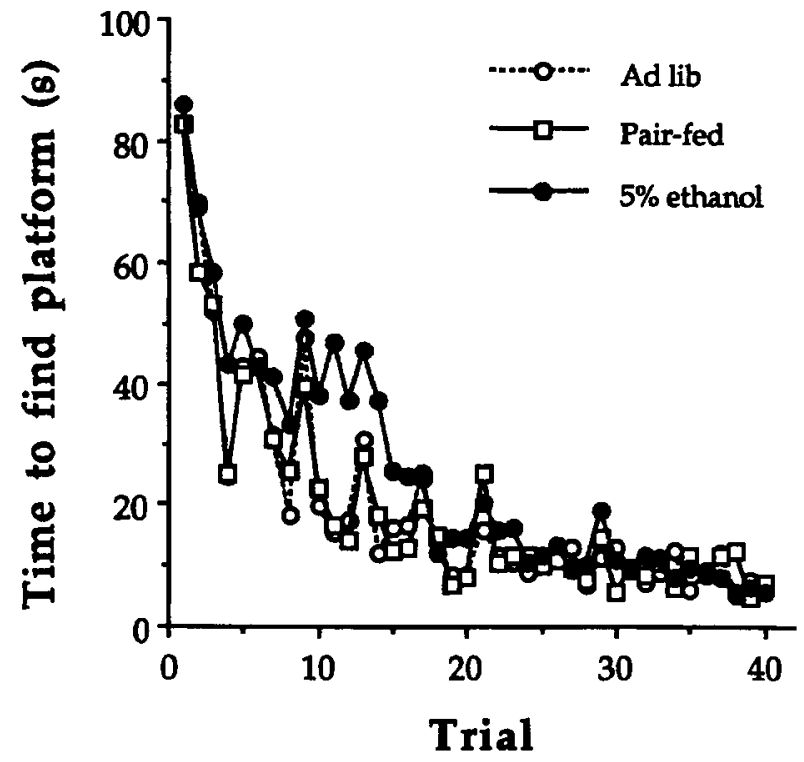

Figure 1. Mean latency for the rats of the three groups to find the hidden platform on each trial during fixed hidden platform training.

trials of each day (Figure 2C). This was especially apparent near the end of training. Figure 2 presents for each maternal diet condition the mean time to find the hidden platform averaged over the first and last 3 days of training. A repeated measures analysis of variance (ANOVA) over these days, with days and trials as within-subjects factors, revealed a significant improvement across days $[F(1,16)=12.6, p<.005]$, a significant improvement across trials $[F(7,112)=4.2, p<.01]$, and a significant interaction between maternal diet condition, days, and trials $[F(14,112)=2.5, p<.01]$. Post hoc tests were conducted using Fisher's LSD test, with $p<.05$. We found that, on the first block of days, Trial 1 and Trial 2 performance significantly differed only for the ad-lib group. On the last block of days, performance on Trial 1 and Trial 2 differed for both ad-lib and pair-fed groups, but not for the 5\% alcohol-exposed group. It is clear that faster Trial 1 performance and slower Trial 2 performance by the latter group contributed to this interaction. Despite the differences in latencies to find the hidden plat-

Table 1

Comparison of the Liquid Diet, Ethanol Consumption, and Blood Ethanol Concentration in Five-Month-Old Sprague-Dawley Rat Dams and the Effects of Diets on Offspring at Birth

\begin{tabular}{lccccc}
\hline $\begin{array}{c}\text { Diet } \\
\text { Group }\end{array}$ & $\begin{array}{c}\text { Food } \\
\text { Consumed }^{\mathrm{a}}\end{array}$ & $\begin{array}{c}\text { Ethanol } \\
\text { Consumed }\end{array}$ & $\begin{array}{c}\text { Serum } \\
\text { EtOH }^{\mathrm{c}}\end{array}$ & $\begin{array}{c}\text { Litter } \\
\text { Sized }^{\mathrm{d}}\end{array}$ & $\begin{array}{c}\text { Pup } \\
\text { Birth Weight }^{\mathrm{e}}\end{array}$ \\
\hline Ad-lib control & n.a. & n.a. & n.a. & $11.4(+1.1)$ & $6.2(+0.2)$ \\
Pair-fed control & 86.9 & n.a. & n.a. & $10.8(+1.3)$ & $6.3(+0.2)$ \\
5\% EtOH diet & 87.1 & 13.1 & $83.2(+3.1)$ & $10.6(+1.4)$ & $6.2(+0.2)$ \\
\hline
\end{tabular}

Note-n.a., not applicable. aMean milliliters liquid diet consumed/day. bMean grams ethanol consumed/ $\mathrm{kilogram}$ body weight/day. ${ }^{c}$ Mean $(+S E M)$ milligrams ethanol/dl serum, $6 \mathrm{~h}$ after introduction of the feeding tubes. dMean $(+S E M)$ number of live births/litter. eMean $(+S E M)$ pup birth weight in grams. 

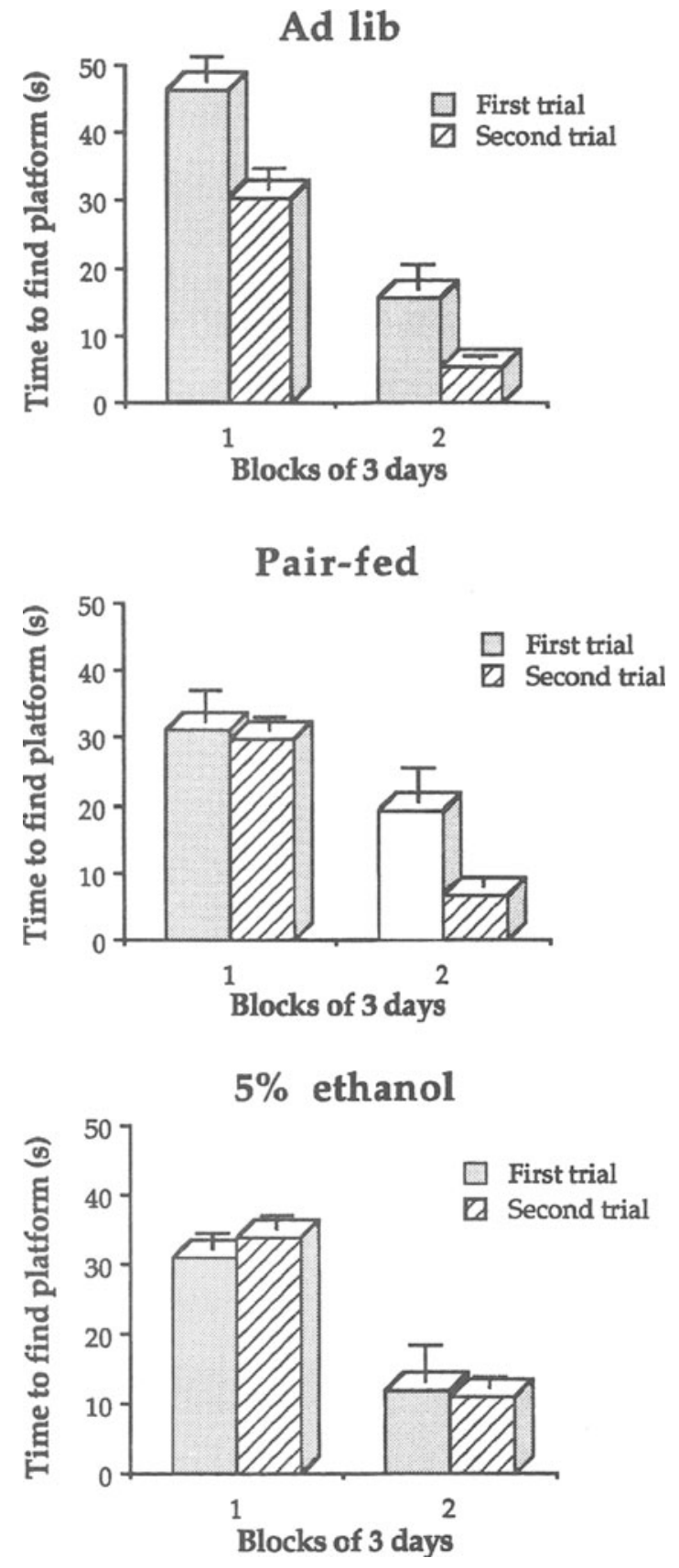

Figure 2. Mean latency $(+S E M)$ to find the hidden moving platform for the first and second daily trials averaged over the first block of 3 days and the last block of 3 days.

form, on the no-platform probe trial, there were no differences among the groups $[F(2,16)=1.1, p<.8]$. The proportions of swimming distances in the correct quadrant were $50.5 \%, 53.3 \%$, and $52.5 \%$ for the ad-lib, pairfed, fetal-alcohol-exposed groups, respectively.

Task 3: Cue-place competition task. The rats of all three groups showed improvements in latency to find the visible platform and the hidden platform (see Figure 3). We conducted two ANOVAs on the acquisition data: one on latency to find the visible platform, and the other on latency to find the hidden platform. The main effect of maternal diet condition on time to find the visible platform was not significant $[F(2,33)=1.0, p<.4]$, but there was a significant improvement across days $[F(8,264)=$ $31.8, p<.0001]$. There was a similar pattern for swimming to the hidden platform during training. The maternal diet groups did not differ $[F(2,33)=0.2, p<.9]$, but they all improved significantly across trial blocks $[F(2,66)=13.7, p<.0002]$.

Despite similar performance measured by time to find the visible or hidden platform during acquisition, the groups showed rather different behavior on the competition test when they were, for the first time, confronted with a choice between swimming to the visible platform located in a new position in the pool and swimming to the place where the platform had been located on every prior trial. For the ad-lib group, 7 of 12 rats swam directly to the cue, ignoring the previously correct place in the pool; for the pair-fed group, this number was 6 of 10 (see Figure 4). In contrast, fully 12 of 14 of the fetalalcohol-exposed rats swam to the cue, ignoring place. The division of cue- versus place-preferring rats was not significantly different from random for the ad-lib group $\left(\chi^{2}=0.33, p=.56\right)$ or for the pair-fed group $\left(\chi^{2}=0.40\right.$, $p=.53$ ). In contrast, the preference for swimming to the cue by fetal-alcohol-exposed group was statistically significant $\left(\chi^{2}=7.14, p<.01\right)$. The swim path of each rat during the place versus cue probe trial is show in Figure 5.

\section{DISCUSSION}

Consistent with earlier reports by Gianoulakis (1990), prenatal exposure to ethanol can produce a long-lasting deficit in spatial learning, as measured by Morris water

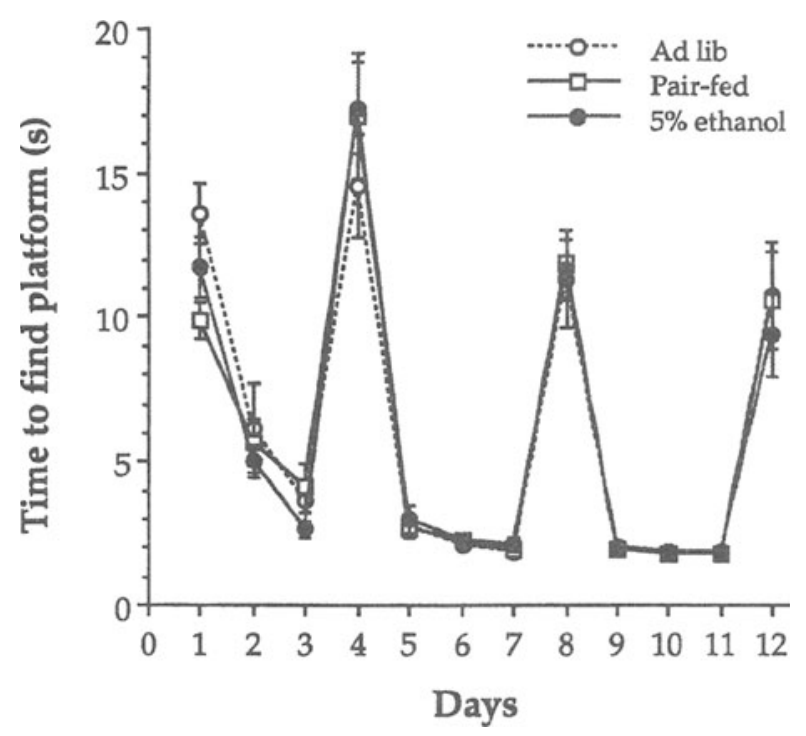

Figure 3. Mean time $(+S E M)$ to find the platform during acquisition of navigation to the visible platform (Days $1,2,3,5,6,7$, 9, 10, and 11) and the hidden platform (Days 4, 8, and 12). 


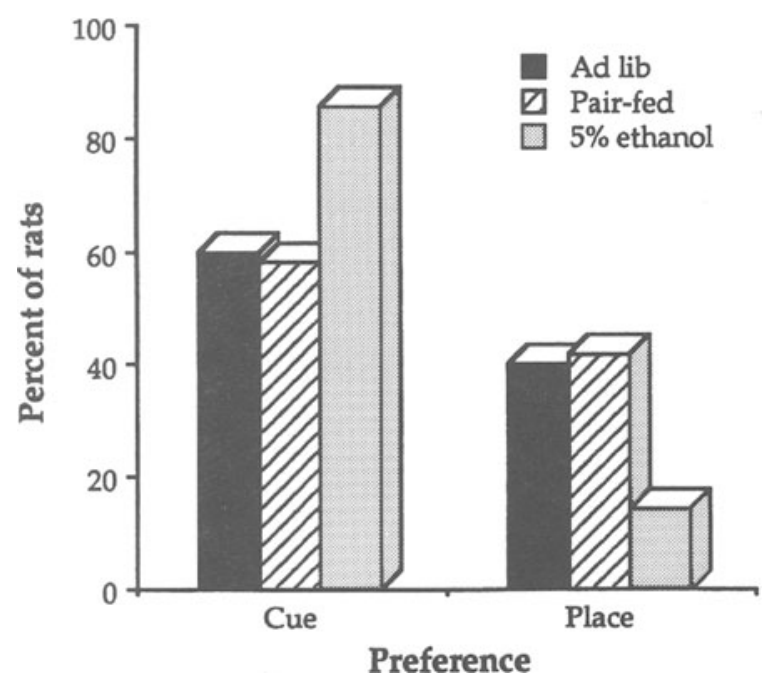

Figure 4. Percentage of rats in the three maternal diet conditions who went directly to the visible platform, preferring not to swim to the previously correct goal location, during the cue versus place competition test on Day 13.

task performance. Unlike Blanchard et al. (1990) and Blanchard et al. (1987), we found that spatial learning can be affected well into adulthood. It is important to note, however, that in the relatively simple fixed hidden platform version of the Morris water task, we were unable to detect a statistically reliable learning deficit in adult offspring of alcohol-drinking dams. When we increased the demands on the rat's place-learning system by requiring the learning of a new hidden goal location each day (Task 2) or by explicitly arranging for a competition between local cue and place information (Task 3 ), we uncovered a relatively subtle but highly reliable deficit in performance. It is important to note that the cue versus place competition task does not explicitly involve an increased task demand per se, but it does increase the burden on a place memory system because the available associative strength accruing on each trial must be divided between the distal spatial cues and the local landmark cue.

Our finding of no deficit in the standard Morris water task but significant deficit in the more demanding versions is not inconsistent with Blanchard et al.'s (1990) conclusion that the spatial deficit may be subtle--our data do count against the suggestion that the deficits are always temporary. Direct comparisons among the present results and those of Blanchard et al. (1990) are made difficult because of the clear differences in alcohol exposure procedures and the age of testing of animals. However, we offer that it may be generally the case that increasing the demands on cognitive systems in rats exposed to moderate prenatal levels of alcohol reveals deficits that may otherwise go undetected.

For a variety of reasons, it is tempting to view these results as symptomatic of hippocampal dysfunction, an idea in line with the conclusions of a recent review of prenatal alcohol effects by Berman and Hannigan (2000). Indeed, disruption of hippocampal function is clearly a possible cause of the spatial learning impairment. Several facts suggest the hippocampal circuitry has been affected into adulthood by fetal alcohol exposure: (1) Induction of LTP at perforant path-dentate synapses is impaired in rats prenatally exposed using our exposure paradigm (Sutherland et al., 1997); (2) long-lasting potentiation of release of radio-labeled aspartate from hippocampal slices induced by high-frequency electrical stimulation is impaired (Savage, Cruz, Duran, \& Paxton, 1998); (3) NMDA receptor binding (Savage et al., 1991) and metabotropic glutamate receptor-stimulated PI hydrolysis (Queen et al., 1993) are abnormal; and (4) hippocampal levels of phospholipases (Allan et al., 1997), protein kinase $\mathrm{C}$, and GAP-43 phosphorylation (PerroneBizzozero et al., 1998), which are thought to participate
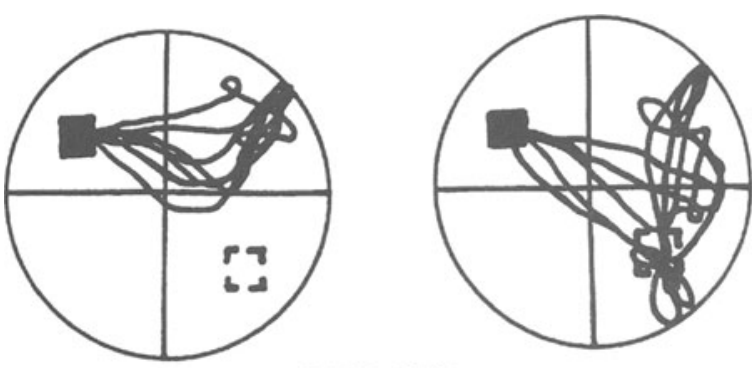

PAIR-FED
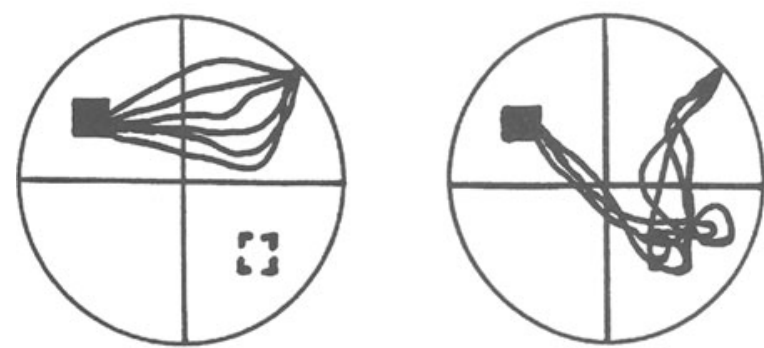

5\% ETHANOL

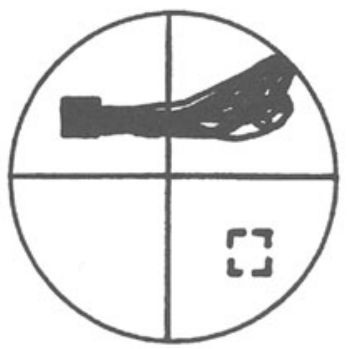

CUE LEARNERS

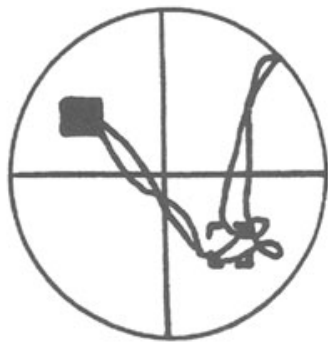

PLACE LEARNBRS
Figure 5. Swim paths of all rats of all conditions during the cue versus place competition test. The solid square indicates the location of the visible platform on the probe trial; the open square indicates the platform location during all training trials. 
in the molecular cascade underlying synaptic plasticity, are chronically reduced by fetal alcohol exposure. The fact that spatial learning is abnormal in adult offspring is certainly consistent with the hypothesis that these neurochemical deficits are involved in producing some of the behavioral abnormalities associated with prenatal alcohol exposure.

Some notes of caution are in order. It is by no means clear that spatial learning is an especially sensitive index of prenatal alcohol cognitive dysfunction. A broad base of cognitive/learning assessments of adult offspring after prenatal exposure is not now in hand. Kim et al. (1992) have shown that prenatal alcohol exposure does not affect object recognition memory measured using a delayednonmatching-to-sample task in rats, even with relatively long delay intervals. Furthermore, clear effects of prenatal exposure can be found in many brain areas other than hippocampal formation, and dysfunctions in many of these circuits could adversely affect cognition or learning, even spatial learning. It could well be that the neurochemical abnormalities that are produced by the moderate prenatal exposure paradigm that we have used are widely distributed throughout the cerebral cortex. Another consideration is the nature of the deficit shown by the alcoholexposed rats in the moving platform task. If their deficit was unequivocally a pure learning effect, then the only abnormality in their performance should have been slower latencies to find the platform on the second and later trials each day. Instead, we found a trend in this direction, but, in addition, these rats showed a trend to be faster in finding the platform on Trial 1. One intriguing possibility is that normal rats are slow to find the platform on the first trial each day in part because they are searching for it in yesterday's location. Prenatally exposed rats may have a weaker memory for the previous location, or they may show less persistence in their search, thus their latencies on Trial 1 may benefit from spending less time in the old correct locations. The idea that they may show a lower level of persistence in their search is not supported by our observations of similar search patterns among groups on no-platform probe trials in Tasks 1 and 2. Finally, our conclusions are limited to prenatally exposed male offspring only; clearly, there could be an important interaction between alcohol exposure and gender of offspring (Kelly, Goodlett, Hulsether, \& West, 1988).

The value associated with better characterization of the cognitive abnormalities, neurochemical changes, and neuroanatomical distribution of effects associated with prenatal alcohol exposure will be found in the possibility of developing and evaluating rational treatment approaches to reverse some of these long-lasting deleterious consequences.

\section{REFERENCES}

Abel, E. L. (1984). Prenatal effects of ethanol. Drug \& Alcohol Dependence, 14, 1-10.

Allan, A. M., Caldwell, K. K., \& Savage, D. D. (1997). Effects of prenatal ethanol exposure on phospholipase $\mathrm{C}$ and $\mathrm{A}_{2}$ in rat hippo- campus and medial frontal cortex. Alcoholism: Clinical \& Experimental Research, 21, 1534-1541.

Allan, A. M., Wu, H., Paxton, L. L., \& Savage, D. D. (1998). Prenatal ethanol exposure alters the modulation of the gamma-aminobutyric acid Al receptor-gated chloride ion channel in adult rat offspring. Journal of Pharmacology \& Experimental Therapeutics, 284, 250-257.

AuER, R. N., JENSEN, M. L., \& Whishaw, I. Q. (1989). Neurobehavioral deficit due to ischemic brain damage limited to half of the CAl sector of the hippocampus. Journal of Neuroscience, 9, 1641-1647.

Berman, R. E., \& HaNNigan, J. H. (2000). Effects of prenatal alcohol exposure on the hippocampus: Spatial behavior, electrophysiology, and neuroanatomy. Hippocampus, 10, 94-110.

Blanchard, B. A., Pilati, M. L., \& Hannigan, J. H. (1990). The role of stress and age in spatial navigation deficits following prenatal exposure to ethanol. Psychobiology, 18, 48-54

Blanchard, B. A., Riley, E., \& Hannigan, J. H. (1987). Deficits on a spatial navigation task following prenatal exposure to ethanol. Neurotoxicology \& Teratology, 9, 253-258.

Castro. C. A., Silbert, L. H., McNaughton, B. L.. \& Barnes, C. A. (1989). Recovery of spatial learning deficits after decay of electrically induced synaptic enhancement in the hippocampus. Nature, 342, 545-548.

Centers for Disease Control and Prevention. (1994). Frequent alcohol consumption among women of childbearing age-Behavioral risk factor surveillance system, 1991. Journal of the American Medical Association, 271, 1820-1821.

Clarren, S. K., \& Smith, D. W. (1978). The fetal alcohol syndrome. New England Journal of Medicine, 298, $1063-1067$.

CONRY, J. (1990). Neuropsychological deficits in fetal alcohol syndrome and fetal alcohol effects. Alcoholism: Clinical \& Experimental Research, 14, 650-661.

Farr, K. L.. Montano. C. Y.. Paxton, L. L., \& Savage. D. D. (1988) Prenatal ethanol exposure decreases hippocampal $3 \mathrm{H}$-vinylidene kainic acid binding in 45-day-old rats. Neurotoxicology \& Teratology, 10, 563-568.

Gianoulakis, C. (1990). Rats exposed prenatally to alcohol exhibit impairment in spatial navigation test. Behavioural Brain Research, 36. 217-228.

Hanson, J. W., Streissguth, A. P., \& Smith, D. W. (1978). The effects of moderate alcohol consumption during pregnancy on fetal growth and morphogenesis. Journal of Pediatrics, 92, 457-460.

JACOBSON, J. L., JACOBSON, S. W., \& SOKOL. R. J. (1994). Effects of fetal alcohol exposure on infant reaction-time. Alcoholism: Clinical \& Experimental Research, 18, 1125-1132.

Jacobson. J. L., Jacobson, S. W.. Sokol., R. J., Martier. S. S., Ager, J. W., \& Kaplan-Estrin, M. G. (1993). Teratogenic effects of alcohol on infant development. Alcoholism: Clinical \& Experimental Research, 17, 174-183.

JONES, K. L., \& SMith. D. W. (1973). Recognition of the fetal alcohol syndrome in early infancy. Lancet, 2, 99-101.

Jones, K. L., Smith, D. W., Ulleland, C. N., \& Streissguth. A. P. (1973). Patterns of malformation in offspring of chronic alcoholic mothers. Lancet, 1, 1267-1271.

Kelly, S., Goodlett. C. R., Hulsether, S.. \& West, J. (1988). Impaired spatial navigation in adult female but not adult male rats exposed to alcohol during the brain growth spurt. Behavioural Brain Research, 27, 247-257.

Kim, C. K., Kalynchuk. L. E., Kornecook, T. J., Redil, V. A., MCIntyre, C. P., Mumby, D. G., Pinel, J. P. J., \& WeinberG. J. (1992). Prenatal ethanol exposure and nonspatial versus spatial learning/ memory in rats. Society for Neuroscience Abstracts, 23, 1237.

Lemoine, P., Haronsseau, H., Borteryu. J. P., \& Menuet, J. C. (1968). Les enfants des parents alcooliques: Anomalies observées à propos de 127 cas [Children of alcoholic parents: Abnormalities observed on the basis of 127 cases]. Ouest Medecine, 25, 476-482.

Mattson, S. N., Riley, E. P., Gramling, L., Delis, D. C., \& Jones, K. L. (1997). Heavy prenatal alcohol exposure with or without physical features of fetal alcohol syndrome leads to IQ deficits. Journal of Pediatrics, 131, 718-721.

McDonald, R. J., \& White, N. M. (1994). Parallel informationprocessing in the water maze: Evidence for independent memory sys- 
tems involving dorsal striatum and hippocampus. Behavioral \& Neural Biology, 61, 260-270.

MiLnER, B. (1965). Visually-guided maze learning in man: Effects of bilateral hippocampal, bilateral frontal, and unilateral cerebral lesions. Neuropsychologia, 6, 215-234.

MORRIS, R. G. M. (1981). Spatial localization does not require the presence of local cues. Learning \& Motivation, 12, 239-261.

Morris, R. G. M., ANderson, E., LyNCH, G. S., \& BAUdRY, M. (1986). Selective impairment of learning and blockade of long-term potentiation by an $\mathrm{N}$-methyl-D-aspartate receptor antagonist, AP5. Nature, 319, 774-776.

Morris, R. G. M., Garrud, P., Rawlins, J. N. P., \& O’Keefe, J. (1982). Place navigation impaired in rats with hippocampal lesions. Nature, 297, 681-683.

Perrone-Bizzozero, N. I., Isaacson, T. V., Keidan, G., Eriqat, C., Meiri, K. F., Savage, D. D., \& Allan, A. M. (1998). Prenatal exposure decreases GAP-43 phosphorylation and protein kinase $\mathrm{C}$ activity in the hippocampus of adult rat offspring. Journal of Neurochemistry, 71, $2104-2111$.

Queen, S. A., Sanchez, C. F., Lopez, S. R., Paxton, L. L., \& Savage, D. D. (1993). Dose and age dependent effects of prenatal ethanol ex posure on hippocampal metabotropic-glutamate receptor-stimulated phosphoinositide hydrolysis. Alcoholism: Clinical \& Experimental Research, 17, 887-893.

Savage, D. D., Cruz, L. L., Duran, L. M., \& Paxton, L. L. (1998). Prenatal ethanol exposure diminishes activity-dependent potentiation of amino acid transmitter release in adult rat offspring. Journal of Pharmacology \& Experimental Therapeutics, 284, 250-257.

Savage, D. D., Montano, C. Y., Otero, M. A., \& Paxton, L. L. (1991). Prenatal ethanol exposure decreases hippocampal NMDAsensitive $[3 \mathrm{H}]$-glutamate binding site density in 45 -day-old rat. $\mathrm{Al}$ cohol, 8, 193-201.

Shaywitz, S. F., Cohen, D. J. \& Shaywitz, B. A. (1980). Behavior and learning difficulties in children of normal intelligence born to alcoholic mothers. Journal of Pediatrics, 96, 978-982.

SkElton, R. W., \& MCNAMARA, R. K. (1992). Bilateral knife cuts to the perforant path disrupt spatial learning in the Morris water maze Hippocampus, 2, 73-80.

SQuiRE, L. (1992). Memory and the hippocampus: A synthesis from findings with rats, monkeys, and humans. Psychological Review, 99 , 195-231.
Streissguth, A. P., Aase, J. M., Clarren, S. K., Randels, S. P., LaDue, R. A., \& SMith, D. F. (1991). Fetal alcohol syndrome in adolescents and adults. Journal of the American Medical Association, 265, 1961-1967.

Streissguth, A., Barr, H., \& Sampson, P. (1990). Moderate prenatal alcohol exposure: Effects on child I.Q. and learning problems at age 71/2 years. Alcoholism: Clinical \& Experimental Research, 14, 662-669.

Sutherland, R. J., Dringenberg, H. C., \& Hoesing, J. M. (1993). Induction of long-term potentiation at perforant path dentate synapses does not affect place learning or memory. Hippocampus, 3, 141-147.

Sutherland, R. J., Hoesing, J. M., \& Whishaw, I. Q. (1990). Domoic acid, an environmental toxin, produces hippocampal damage and severe memory impairment. Neuroscience Letters, 120, 221-223.

Sutherland, R. J., Kolb, B., \& Whishaw, I. Q. (1982). Spatial mapping: Definitive disruption by hippocampal and frontal cortex damage in the rat. Neuroscience Letters, 31, 271-276.

Sutherland, R. J., McDonald, R. J., \& Savage, D. D. (1997). Prenatal exposure to moderate levels of ethanol can have long-lasting effects on hippocampal synaptic plasticity in adult offspring. Hippocampus, 7, 232-238.

Sutherland, R. J., \& Rodriguez, A. J. (1989). On the role of the fornix and related subcortical structures in spatial learning and memory. Behavioural Brain Research, 32, 265-277.

Sutherland, R. J., Whishaw, I. Q., \& Kolb, B. (1983). A behavioural analysis of spatial localization following electrolytic, kainate-, or colchicine-induced damage to the hippocampal formation in the rat. Behavioural Brain Research, 7, 133-153.

WHISHAW, I. Q. (1985). Formation of place learning-set by the rat: A new paradigm for neurobehavioral studies. Physiology \& Behavior, 35 , 139-143.

WHISHAW, I. Q. (1987). Hippocampal, granule cell and CA3-4 lesions impair formation of a place learning-set in the rat and induce reflex epilepsy. Behavioural Brain Research, 24, 59-72.

Zola-Morgan, S.. SQuire, L., \& Amaral, D. (1986). Human amnesia and the medial temporal lobe region: Enduring memory impairment after a bilateral lesion limited to CAl field of the hippocampus. Journal of Neuroscience, 6, 2950-2967.

(Manuscript received April 5, 2000; revision accepted for publication May 31, 2000.) 